\title{
Rationale and Design of a Prospective, Multicenter, Observational Study Evaluating Iron Deficiency in Patients Hospitalized for Heart Failure (FERIC-RO)
}

\author{
Elena Laura Antohi ${ }^{1}$, Gabriel Tatu Chitoiu ${ }^{2}$, Andrew P Ambrosy ${ }^{3}$, Ioan \\ M Coman ${ }^{4}$, Dragos Vinereanu ${ }^{5}$, Sean P Collins ${ }^{6}$, Crina Sinescu ${ }^{7}$, Serban \\ Mihaileanu $^{8}$, Peter S Pang ${ }^{9}$, Javed Butler ${ }^{10}$, Ovidiu Chioncel ${ }^{1 *}$ \\ 1. Institute of Emergency for Cardiovascular Diseases 'Prof. C.C. Iliescu' \\ 2. Emergency Hospital Floreasca, Cardiology Department, Bucuresti, Romania
}

3. Duke University Medical Center, Durham, NC, US

4. Institute of Emergency for Cardiovascular Diseases 'Prof. C.C. Iliescu', University of Medicine and Pharmacy Carol Davila, Bucuresti, Romania

5. Universitary Hospital, University of Medicine and Pharmacy Carol Davila, Bucuresti, Romania 6. Department of Emergency Medicine, Vanderbilt University,

Nashville, Tennessee, US

7. Bagdasar Emergency Hospital, University of Medicine and Pharmacy Carol Davila, Bucuresti, Romania

8. Institut Mutualiste Montsouris, Paris, France

9. Department of Emergency Medicine, Indiana University School of Medicine, Indiana, US

10. Department of Medicine, University of Mississippi School of Medicine, Jackson, MI, USA

\begin{abstract}
Introduction: Several landmark studies, which enrolled heart failure (HF) patients who were ambulatory at the time of inclusion, identified iron deficiency (ID) as an important therapeutic target: intravenous iron administration with ferric carboxymaltose (FCM) improves morbidity, exercise capacity, and quality of life in patients with $H F$ and reduced EF (HFrEF). However, there is still limited knowledge about ID prevalence during hospitalization for Worsening Chronic HF (WCHF) and about the relationship between ID during hospitalization and post-discharge outcomes. Although previous studies documented ID as an independent risk factor for poor outcomes in $H F r E F$, its prognostic significance in $H F$ patients with $E F>40 \%$ remains unclear.

Method and Results: The FERIC-RO study is a prospective, multicenter, observational study with longitudinal follow up, conducted in 9 Romanian hospitals that will include 200 consecutive patients admitted for worsening
\end{abstract}

*Corresponding author: Ovidiu Chioncel, Institute of Emergency for Cardiovascular Diseases 'Prof. C.C. Iliescu', Bucharest, Romania. E-mail: ochioncel@yahoo.co.uk 
HF. A comprehensive description of the Iron metabolism biomarkers will be performed on discharge and 1-month follow up. The primary endpoint is defined as the prevalence of ID on discharge and 1-month post-discharge, and the secondary endpoints include: all-cause re-hospitalization and all-cause-mortality at 1 and 3 months follow up, and quality of life on discharge and 1-month.

Conclusions: FERIC-RO will provide new evidence about the prevalence and the predictors of ID in patients hospitalized for WCHF regardless of LVEF. Furthermore, the study will explore the relationship between in-hospital ID and post-discharge outcomes. The results of FERIC-RO will thus be highly relevant to the management of patients hospitalized for AHF.

Keywords: heart failure, iron deficiency, clinical studies

Received: 13 $3^{\text {th }}$ May 2018; Accepted: 18 ${ }^{\text {th }}$ June 2018; Published: $24^{\text {th }}$ June 2018

\section{Introduction}

Heart failure (HF) is a syndrome associated with impaired quality of life, functional limitations, high CV morbidity and mortality, and burgeoning direct and indirect costs. (1-4)

As compared to chronic HF patients who benefit from GDMTs, patients hospitalized for acute HF have an unacceptably high outcome rate, either in-hospital and several months post discharge, probably due to the fact that no effective treatments have been implemented in practice over the last decades (5-7). Of patients hospitalized for AHF, $60-70 \%$ are WCHF, patients with previous history of HF hospitalization, while $20-30 \%$ are de novo HF, patients at first HF decompensation. Distinct to patients with de novo HF, those hospitalized for worsening chronic HF have a much higher post-discharge event rate, despite clinical and hemodynamic improvement during hospitalization (8). One major contributing factor to worsening post-discharge outcomes in WCHF is the coexistence of non-cardiac comorbidities (NCC). Particularly, for Romanian patients hospitalized for WCHF, a high prevalence of NCC has been reported (9). Left untreated, many of these NCC contribute to exacerbating the HF process or decreasing the threshold for occurrence of symptoms. Ultimately, this leads to worsen- ing $\mathrm{HF}$ and a subsequent hospitalization. Of particular importance among NCC is the presence of iron deficiency (ID), which is a major factor in the pathogenesis of anemia, but it is also a distinct condition with serious clinical consequences (e.g. impaired exercise capacity, quality of life) and poor prognosis in HFrEF patients. Iron is a micronutrient essential for cellular energy and metabolism, necessary for maintaining body homoeostasis. It is essential for mitochondrial respiration and is involved in the adaptation to acute and chronic increases in myocardial workload (10).

For chronic HF patients, previous studies demonstrate ID as an important therapeutic target: intravenous iron administration restores inotropic reserve in animal models and improves morbidity and symptoms in clinical studies enrolling chronic HFrEF patients $(10,11)$. However, although previous studies documented ID as an independent risk factor for poor outcomes in HF-rEF, its prognostic significance in HF patients with $\mathrm{EF}>40 \%$ remains unclear.

The prevalence of ID in HF patients has been reported to range from 30 to $50 \%$, but studies were conducted in outpatient setting in a relative stable condition (12) ID includes absolute ID defined as serum ferritin $<100 \mu \mathrm{g} / \mathrm{L}$ and relative ID, defined as serum ferritin $100-299 \mu \mathrm{g} / \mathrm{L}$ and a transferrin saturation (TSAT) $<20 \%$ accord- 
ing to the 2016-ESC-HF Guidelines (13). These levels have a good sensitivity and specificity to identify the patients who will benefit from iron therapy $(13,14)$. However, as iron is regulated across circulating, functional, and storage components, several other markers emerged to further characterize the deficit and differentiate between the truly depleted iron and the defectively mobilized iron (i.e. anemia of chronic disease or inflammation as it is often the case in HF) (15, 16). An increase in the serum value of soluble transferrin receptor 1 (sTfR) can be used to identify ID more accurately when ferritin and TSAT values are divergent, and may predict hematological response to treatment. (17) Hepcidin is an essential regulatory hormone for iron metabolism by interacting with its receptor ferroportin and inducing hypoferremia, but its metabolism is still unpredictable and its role in the pathogenesis of ID seen in HF has not been sufficiently documented (18).

To date, numerous HF studies suggest that in chronic settings, ID is highly prevalent, associated with adverse outcomes, and responsive to IV repletion, independent of anemia status. However, in contrast to chronic HF, numerous knowledge gaps remain regarding ID prevalence and Iron metabolism in the acute HF setting. Targeting ID may be a key treatment target. In a recent study enrolling patients with AHF, the reported prevalence of ID was $68.6 \%$ in men and even higher (75.3\%) in women. (19) Of note, ID in this acute setting was not related to HF severity, emphasizing the need for iron deficiency screening at the time of hospitalization for all patients with worsening HF (19). To note, although in chronic settings ID was associated with poor outcomes, very scarce information exists concerning the relationship between ID evaluated during hospitalization and post-discharge outcomes.

The FERIC-RO (FiER deficit in Insuficienta Cardiaca in ROmania) study aims to accurately describe the epidemiology, clinical characteristics, and mechanisms of ID in patients hospitalized for chronic worsening HF. The main objectives of FERIC-RO are: 1 . to determine the prevalence of ID on discharge and 1-month, 2. to identify predictors of ID, 3 . to evaluate the association between ID and clinical outcomes. The study protocol allows identification and complete characterization of alteration in iron homeostasis in patients hospitalized for chronic worsening HF for any level of LVEF and will bridge knowledge gaps regarding the relationship between in-hospital ID and post-discharge outcomes.

\section{Methods}

\section{Study Overview}

The FERIC-RO study is a prospective, multicenter, observational study with longitudinal follow up, conducted in 9 Romanian hospitals, which will include 200 consecutive HF patients. At the time of hospital admission, eligible patients must have a primary diagnosis of chronic worsening HF regardless of LVEF. Inclusion and exclusion criteria are summarized in Table 1. Follow-up visits will be performed at 1 and 3 months post-discharge and study patients will return for follow up to the same hospital where they were enrolled. Telephone follow-up will collect additional information about vital status at 6 and 12 months post-discharge as detailed in Figure 1.

\section{Study endpoints}

The primary clinical endpoint is defined as the prevalence of ID on discharge and 1-month post discharge.

The secondary endpoints include: all cause re-hospitalization and ACM events at 1 and 3 months follow up, and quality of life on discharge and 1-month. 
Table 1. Inclusion and exclusion criteria in FERIC-RO study.

A.

\begin{tabular}{ll}
\hline Inclusion criteria & Exclusion criteria \\
\hline Informed signed consent. & $\begin{array}{l}\text { Patients with prior oral or intravenous iron supplementation within the past } \\
3 \text { months. }\end{array}$ \\
\hline Age above 18 years. & $\begin{array}{l}\text { Suspected acute coronary syndrome on presentation (i.e. defined as angina } \\
\text { equivalent in the setting of electrocardiographic signs of ischemia or pos- } \\
\text { itive biomarkers). }\end{array}$ \\
$\begin{array}{l}\text { Patients who present signs and Cardiogenic shock at presentation (i.e. defined as a systolic blood pressure } \\
\text { symptoms of HF with need of hos- } \\
\text { pitalization and urgent/emergent IV } \\
\text { therapies. }\end{array}$ \\
$\begin{array}{l}\text { Previous HF hospitalization in the De novo HF. } \\
\text { last } 12 \mathrm{months} \text {. }\end{array}$ \\
$\begin{array}{l}\text { BNP }>300 \mathrm{pg} / \mathrm{ml} \text { or NTproBNP } \text { Conditions which will impede the full participation in the study such as } \\
>1000 \mathrm{pg} / \mathrm{ml} \text { at the time of hospital } \\
\text { admission. }\end{array}$ \\
\hline
\end{tabular}

B. discharged alive after hospitalization for AHF

Abbreviations: $\mathrm{HF}=$ heart failure, $\mathrm{IV}=$ intravenous, $\mathrm{BNP}=$ brain natriuretic peptide, $\mathrm{NT}$-proBNP= $\mathrm{N}$-terminal prohormone $\mathrm{BNP}$.

\section{Protocol}

Written informed consent was obtained from each patient. The protocol was conducted in accordance with the Declaration of Helsinki and Guidelines on Good Clinical Practice, and approved by a local ethics committee. The physicians will continue the treatment of the patients according to their daily practice. No specific analyses or treatments will be prescribed as part of this study. The patient's epidemiological characteristics on inclusion, including demography, vitals, clinical presentation, medical history, associated non-cardiac comorbidities (NCC), medical triage, prior treatments, as well as in-hospital management and complications will be recorded (Figure 1). BNP/NT-pro-BNP will be collected on admission and discharge. A comprehensive evaluation of anemia and ID will be performed on discharge and 1-month post-discharge (Table 2). Quality of life will be evaluated by Kansas City Cardiomyopathy Questionnaire (KQOL) questionnaire on discharge and at 1 month.
A central laboratory will receive the peripheral blood samples on discharge and at 1 month for determination of ID parameters and inflammatory parameters (Table 2). A subset of patients (i.e. $\sim 10 \%$ ) will have additional biomarkers drawn including TNF-alpha, interleukin-6, and hepcidin. The blood samples will be collected and centrifuged after $30 \mathrm{~min}$ and transferred to the central laboratory on the same day.

The analysis of blood samples on admission will be performed at the local laboratory and will include routine blood assessment, as indicated in Table 2.

\section{Statistics}

Due to the observational nature of this study, no formal sample size calculation has been performed. A consecutive cohort of 200 AHF patients represents the study population.

The primary endpoint (prevalence of ID on discharge and at 1 month) will be evaluated as proportion of patients with this characteristic. Based on previous results (19-24), the expected 


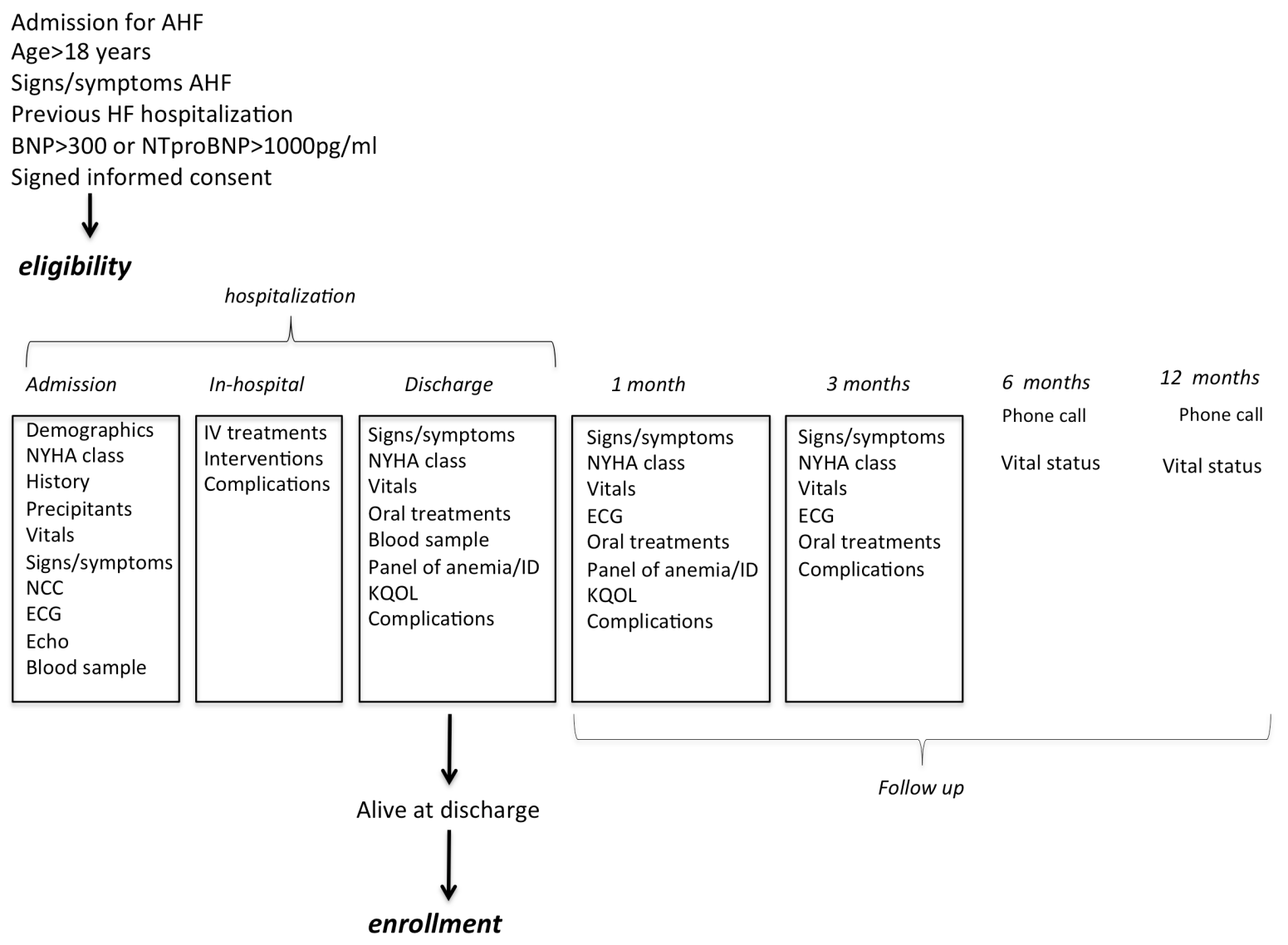

Fig. 1. Schedule of assessments and procedures during study follow up.

proportion for the primary endpoint occurrence is $55-70 \%$.

Subgroup analyses will evaluate the prevalence of ID in anemic and non-anemic patients as well as the potential effects of age, gender, LVEF, and prior history of HF. A stepwise logistic regression model will be used to select the subgroup of parameters significantly associated with iron deficiency in the study population, both on discharge and at 1 month.

Absolute frequencies and proportions of patients who died or were hospitalized at 1 and 3 months will be provided for both the ID and non-ID groups. Kaplan-Meier estimates of the survival functions will be plotted respective of presence or absence of ID on discharge.
Independent predictors for the secondary endpoints, deaths and re-admissions at 3 months post discharge, will be identified using a multivariate logistic regression model. Multivariate analysis will be performed using forward conditional regression, whereby variables will be removed from the equation after each iteration if the type I error rate was greater than $10 \%$ and retained in the final equation if $\mathrm{P} \leq$ .05. All statistical analyses will be two-tailed, with a threshold for significance set at a $\mathrm{P}$ value of 0.05 or less. All final analyses will be conducted using SPSS software, version 16 (SPSS Inc, Chicago, IL). 
Table 2. Evaluation of anemia and ID indexes during the study follow-up

\begin{tabular}{lll}
\hline Admission & Discharge & 1 Month \\
\hline Hemoglobin & Hemoglobin & Hemoglobin \\
\hline Ht & Ht & Ht \\
\hline Mean cell volume & Mean cell volume & Mean cell volume \\
Mean cell hemoglobin & Mean cell hemoglobin & Mean cell hemoglobin \\
MCHC & MCHC & MCHC \\
\hline Red cell distribution width & Red cell distribution width (\%) & Red cell distribution width \\
White cell count & White cell count & White cell count \\
\hline Platelet count & Platelet count & Platelet count \\
\hline ESR & Vitamin B12 & - \\
\hline Fibrinogen & Folate & - \\
\hline NTproBNP & Iron & Iron \\
Troponine I & Ferritin & Ferritin \\
Creatinine & TSAT & TSAT \\
BUN & TIBC & TIBC \\
Na & sTfR & sTFR \\
K & & \\
Cholesterol & & \\
Albumine & IL6 & C reactive protein \\
\hline- & Hepcidin & - \\
\hline- & TNF & - \\
\hline- & K &
\end{tabular}

Abbreviations: $\mathrm{Ht}=$ hematocrit, $\mathrm{Na}=$ sodium, $\mathrm{K}=$ potassium, $\mathrm{ESR}=$ erythrocite sedimentation rate, $\mathrm{MCHC}=$ mean corpuscular hemoglobin concentration, TSAT $=$ transferrin saturation, $\mathrm{TIBC}=$ total iron binding capacity(TSAT calculated as $100 \mathrm{Xserum}$ ferritin/TIBC), sTfR =soluble transferrin receptor $1, \mathrm{IL} 6=$ interleukin $6, \mathrm{TNF}=$ tumor necrosis factor

\section{Discussion}

Clinical outcomes for patients with WCHF are exceptionally poor, and, to date, in-hospital treatment for these patients remains limited to decongestion and the treatment of cardiac and non-cardiac co-morbidities.

Anemia (defined according to the WHO definition as $<12 \mathrm{~g} / \mathrm{dl}$ in women and $<13 \mathrm{~g} / \mathrm{dl}$ in men) is a common NCC in HF patients $(25,26)$. However, the relationship between anemia and outcomes has been inconsistent and may largely be confounded by renal dysfunction, congestion status, and ID. There are no data to demonstrate that targeting anemia improves clinical outcomes (i.e. RED-HF) (27). In contrast, there are data that IV FCM improves QoL and functional status in iron deficient patients with HFrEF enrolled in several CV outcomes trials $(28,29)$.

The prevalence of ID anemia (whether it is iron depleted or iron defectively mobilized) has been shown to correlate with severity of HF in chronic settings (30). Chronic iron deficiency, by itself, may cause ultrastructural alterations in myocytes (31). Recent evidence suggests ID in HFrEF patients may be associated with impaired exercise capacity, more severe disease (higher NYHA class), and poorer outcomes (32-34). Even in the absence of anemia, ID is a potent substrate for dyspnea and exercise intolerance and identifies patients at a heightened risk for mortality $(31,35,36)$, suggesting that ID should be regarded as a distinct clinical condition that 
may be an even stronger predictor of outcome than anemia (30).

Body iron metabolism may be abnormal at any level of distribution: circulation, functional, storage and therefore multiple markers are necessary to adequately characterize it (each marker reflects a certain compartment) (30). Studies have also shown an alteration in iron distribution in chronic heart failure (circulating iron is contracted and storage compartment is expanded) (30). Ferritin and hepcidin are acute phase reactants and their changes strongly impact iron homeostasis. It is therefore reasonable to expect differences at sensitive levels in acute setting.

Iron deficit was typically defined as ferritin $<100 u g / 1$, but as this cutoff has limited sensibility, TSAT $<20 \%$ may be a more reliable marker for iron status when inflammation is present, as is often the case in HF patients (reflected by high CRP levels) $(13,37)$. When the two markers have divergent values (high ferritin and low TSAT), sTfR and the derived sTfR/log ferritin index may provide additional information (38). Because sTFR is not influenced by inflammation, its value may help differentiate between chronic disease anemia and isolated iron deficit $(30,37)$. Its increase is an indirect measure of the increase in the transmembrane dimer (TfR) that is overexpressed when cells (especially erythroid progenitor cells) require iron and may thus be a good predictor for red blood cell response to iron therapy (39). Measuring sTfR has previously been questioned as assays from different manufacturers have not been standardized and are not widely available (38). In our study we will use immunonephelometry which has shown a good limit of agreement with other techniques (40).

Hepcidin is a peptide synthetized by the liver and is an iron regulatory hormone, especially in inflammatory status when it is up-regulated (41). IL-6 is the cytokine that increases the expression of the hepcidin gene and thus lowers the bioavailability of iron (42). Since HF is a condition associated with high levels of inflammatory cytokines (like IL-6 and TNF- $\alpha$ ) (43), it would be expected that the plasmatic level of hepcidin increases in decompensated $\mathrm{HF}$, but there are caveats that could impact the results, as diurnal (lower in the morning) and gender (lower in women) variations have been observed (16). To avoid diurnal variations, in the present study, the blood samples on discharge will be collected in all patients in the first hours in the morning, in order to homogenize the results.

After the positive results of the FAIR-HF (Ferinject Assessment in Patients with Iron Deficiency and Chronic Heart Failure) and CONFIRM-HF (Ferric Carboxymaltose evaluation on perFormance in patients with Iron deficiency in combination with chronic Heart Failure) trial, there is a legitimate need to study the iron metabolism of patients with AHF, and a work-up to identify the determinants of ID in the acute setting may prove beneficial $(28,29)$. Secondly, the impact of in-hospital ID, with- or without anemia, on post-discharge outcomes should be evaluated in further research derived from RCTs and observational studies. The results of this type of studies may help to clarify when to start treatment with IV iron relative to the time point of hospitalization.

FERIC-RO is distinct from all other completed RCTs and observational studies by its design looking for in-hospital prevalence of ID in a cohort of patients hospitalized for AHF regardless of the level of LVEF. A major goal of FERIC-RO study is to obtain data under conditions representative of real-world clinical practice and thus generate conclusions that have greater external validity (generalizability) than those derived from conventional RCTs. Inclusion and exclusion criteria were pragmatically chosen to define a study cohort representative of the worsening chronic HF population. The rationale for the selection criteria of the minimum qualifying level 
of BNP/NT-pro-BNP was to make inclusion of patients with non-cardiogenic dyspnea less likely. The primary endpoint has been pragmatically chosen to identify the potential candidates for IV Iron therapy as well as the moment of initiation. In addition, a rigorous investigation of in-hospital and 1-month post-discharge Iron metabolism and predictors of ID is another major strength of this study. The secondary endpoints included all cause re-hospitalization and all cause mortality events at 1 and 3 months follow up. In two trials, EVEREST and ASTRONAUT, which enrolled a similar patient population as FERIC-RO, the reported rate of death or HF hospitalization at 3 months was $20 \%$, and similar outcome rate is expected in the present study $(44,45)$.

These secondary endpoints are clinically relevant, and may be potentially responsive to therapeutic interventions with FCM during hospitalization, and thus important to both patients and healthcare providers.

\section{Conclusion}

Iron metabolism strongly impacts myocardial and skeletal muscle function in HF. ID is common in patients with chronic HF regardless of anemia status and ID should be considered as a distinct comorbidity and specific therapeutic target, since it has a greater predictive value for outcome than the presence of anemia. FERIC-RO may provide further evidence about the prevalence of ID in patients hospitalized for AHF and may elucidate prognostic importance of in-hospital ID for post-discharge outcomes. Furthermore, this acute settings study will provide a complete characterization of iron metabolism biomarkers and their interaction with clinical and biological variables collected during hospitalization for AHF.

\section{Acknowledgements}

The study was an investigator driven initiative, financially supported by Vifor Pharma,
Servier, Novartis and Roche Diagnosis and these entities had no involvement in data collection, analysis, or interpretation and played no role in the preparation of this manuscript.

The authors acknowledge the significant contribution of Prof. Mihai Gheorghiade for the concept and design of this study. We appreciate the continuous scientific inputs given by Claudio Mori for the developing study design and implementation of the study protocol.

\section{Author's contribution:}

E.L.A-drafting the manuscript and data acquisition; G.T.C.-drafting the protocol and critical revision of the manuscript; A.P.A- drafting the protocol and critical revision of the manuscript; I.M.C- drafting the protocol and critical revision of the manuscript; D.V.- drafting the protocol and critical revision of the manuscript; C.S.- drafting the protocol and critical revision of the manuscript; S.P.C.- drafting the protocol and critical revision of the manuscript; P.P.- drafting the protocol and critical revision of the manuscript; S.M.drafting the protocol and critical revision of the manuscript; J.B.- drafting the protocol and critical revision of the manuscript; O.C.- drafting the protocol and critical revision of the manuscript; M.G. and O.C. were Principal Investigators.

\section{Abbreviations}

AHF acute decompensated heart failure, HF heart failure, BNP brain natriuretic peptide, NTproBNP N-terminal prohormone BNP, NYHA New York Heart Association, NCC non cardiac comorbidities, ECG electrocardiogram, Echo echocardiography, IV intravenous, ID iron deficit, KQOL Kansas City Cardiomyopathy Questionnaire,

\section{References}

1. Levy D, Kenchaiah S, Larson MG, Benjamin EJ, Kupka MJ, Ho KK, et al. Long-term trends in the in- 
cidence of and survival with heart failure. $\mathrm{N}$ Engl $\mathrm{J}$ Med. 2002;347(18):1397-402. DOI: 10.1056/NEJMoa020265

2. Stewart S, Jenkins A, Buchan S, McGuire A, Capewell S, McMurray JJ. The current cost of heart failure to the National Health Service in the UK. Eur J Heart Fail. 2002;4(3):361-71. DOI: 10.1016/S13889842(01)00198-2

3. Schaufelberger M, Swedberg K, Koster M, Rosen M, Rosengren A. Decreasing one-year mortality and hospitalization rates for heart failure in Sweden; Data from the Swedish Hospital Discharge Registry 1988 to 2000. Eur Heart J. 2004;25(4):300-7. DOI: 10.1016/j. ehj.2003.12.012

4. Mosterd A, Hoes AW. Clinical epidemiology of heart failure. Heart. 2007;93(9):1137-46. DOI: 10.1136/ hrt.2003.025270

5. Tavazzi L, Senni M, Metra M, Gorini M, Cacciatore G, Chinaglia A, et al. Multicenter prospective observational study on acute and chronic heart failure: one-year follow-up results of IN-HF (Italian Network on Heart Failure) outcome registry. Circ Heart Fail. 2013;6(3):473-81. DOI: 10.1161/CIRCHEARTFAILURE.112.000161

6. Crespo-Leiro MG, Anker SD, Maggioni AP, Coats AJ, Filippatos G, Ruschitzka F, et al. European Society of Cardiology Heart Failure Long-Term Registry (ESCHF-LT): 1-year follow-up outcomes and differences across regions. Eur J Heart Fail. 2016;18(6):613-25. DOI: 10.1002/ejhf.566

7. Hamo CE, Butler J, Gheoghiade M, Chioncel O. The bumpy road to drug development for acute heart failure. European Heart Journal Supplements. 2016;18(suppl_G, 1 November 2016):G19-G32.

8. O'Connor CM, Miller AB, Blair JE, Konstam MA, Wedge P, Bahit MC, et al. Causes of death and rehospitalization in patients hospitalized with worsening heart failure and reduced left ventricular ejection fraction: results from Efficacy of Vasopressin Antagonism in Heart Failure Outcome Study with Tolvaptan (EVEREST) program. Am Heart J. 2010;159(5):841-9 e1.

9. Antohi EL, Dobreanu D, Deleanu D, Tatu-Chitoiu G, Macarie C, Chioncel O. Relation between non-cardiac comorbidities and use of evidence based therapies in patients with in-hospital heart failure-data from SONIC-RO study. Eur J Heart Fail Suppl. 2013;12:S354-S80. 10. Haddad S, Wang Y, Galy B, Korf-Klingebiel M, Hirsch
$\mathrm{V}$, Baru AM, et al. Iron-regulatory proteins secure iron availability in cardiomyocytes to prevent heart failure. Eur Heart J. 2017;38(5):362-72.

11. Jankowska EA, Tkaczyszyn M, Suchocki T, Drozd M, von Haehling S, Doehner W, et al. Effects of intravenous iron therapy in iron-deficient patients with systolic heart failure: a meta-analysis of randomized controlled trials. Eur J Heart Fail. 2016;18(7):786-95. DOI: 10.1002/ejhf.473

12. Anand IS. Anemia and chronic heart failure implications and treatment options. J Am Coll Cardiol. 2008;52(7):501-11. DOI: 10.1016/j.jacc.2008.04.044

13. Wish JB. Assessing iron status: beyond serum ferritin and transferrin saturation. Clin J Am Soc Nephrol. 2006;1 Suppl 1:S4-8. DOI: 10.2215/CJN.01490506

14. Ponikowski P, Voors AA, Anker SD, Bueno H, Cleland JGF, Coats AJS, et al. 2016 ESC Guidelines for the diagnosis and treatment of acute and chronic heart failure: The Task Force for the diagnosis and treatment of acute and chronic heart failure of the European Society of Cardiology (ESC)Developed with the special contribution of the Heart Failure Association (HFA) of the ESC. Eur Heart J. 2016;37(27):2129-200. DOI: 10.1093/eurheartj/ehw128

15. Kalantar-Zadeh K, Rodriguez RA, Humphreys MH. Association between serum ferritin and measures of inflammation, nutrition and iron in haemodialysis patients. Nephrol Dial Transplant. 2004;19(1):141-9. DOI: $10.1093 /$ ndt/gfg493

16. Ganz T, Nemeth E. Hepcidin and iron homeostasis. Biochim Biophys Acta. 2012;1823(9):1434-43. DOI: 10.1016/j.bbamcr.2012.01.014

17. Feelders RA, Kuiper-Kramer EP, van Eijk HG. Structure, function and clinical significance of transferrin receptors. Clin Chem Lab Med. 1999;37(1):1-10. DOI: 10.1515/CCLM.1999.001

18. Divakaran V, Mehta S, Yao D, Hassan S, Simpson S, Wiegerinck E, et al. Hepcidin in anemia of chronic heart failure. Am J Hematol. 2011;86(1):107-9. DOI: 10.1002/ajh.21902

19. Cohen-Solal A, Damy T, Terbah M, Kerebel S, Baguet JP, Hanon O, et al. High prevalence of iron deficiency in patients with acute decompensated heart failure. Eur J Heart Fail. 2014;16(9):984-91. DOI: 10.1002/ ejhf.139

20. Solomon SD, Dobson J, Pocock S, Skali H, McMurray JJ, Granger CB, et al. Influence of nonfatal hos- 
pitalization for heart failure on subsequent mortality in patients with chronic heart failure. Circulation. 2007;116(13):1482-7. DOI: 10.1161/CIRCULATIONAHA.107.696906

21. Jack BW, Chetty VK, Anthony D, Greenwald JL, Sanchez GM, Johnson AE, et al. A reengineered hospital discharge program to decrease rehospitalization: a randomized trial. Ann Intern Med. 2009;150(3):178-87. DOI: 10.7326/0003-4819-150-3-200902030-00007

22. Ross JS, Chen J, Lin Z, Bueno H, Curtis JP, Keenan PS, et al. Recent national trends in readmission rates after heart failure hospitalization. Circ Heart Fail. 2010;3(1):97-103. DOI: 10.1161/CIRCHEARTFAILURE.109.885210

23. Dharmarajan K, Hsieh AF, Lin Z, Bueno H, Ross JS, Horwitz LI, et al. Diagnoses and timing of 30-day readmissions after hospitalization for heart failure, acute myocardial infarction, or pneumonia. JAMA. 2013;309(4):355-63. DOI: 10.1001/jama.2012.216476

24. Go AS, Mozaffarian D, Roger VL, Benjamin EJ, Berry JD, Borden WB, et al. Heart disease and stroke statistics--2013 update: a report from the American Heart Association. Circulation. 2013;127(1):e6-e245. DOI: 10.1161/CIR.0b013e31828124ad

25. Nutritional anaemias. Report of a WHO scientific group. World Health Organ Tech Rep Ser. 1968;405:537.

26. Chioncel O, Vinereanu D, Datcu M, Ionescu DD, Capalneanu R, Brukner I, et al. The Romanian Acute Heart Failure Syndromes (RO-AHFS) registry. Am Heart J. 2011;162(1):142-53 e1.

27. Swedberg K, Young JB, Anand IS, Cheng S, Desai AS, Diaz R, et al. Treatment of anemia with darbepoetin alfa in systolic heart failure. N Engl J Med. 2013;368(13):1210-9. DOI: 10.1056/NEJMoa1214865

28. Anker SD, Comin Colet J, Filippatos G, Willenheimer R, Dickstein K, Drexler H, et al. Ferric carboxymaltose in patients with heart failure and iron deficiency. N Engl J Med. 2009;361(25):2436-48. DOI: 10.1056/ NEJMoa0908355

29. Ponikowski P, van Veldhuisen DJ, Comin-Colet J, Ertl G, Komajda M, Mareev V, et al. Beneficial effects of long-term intravenous iron therapy with ferric carboxymaltose in patients with symptomatic heart failure and iron deficiencydagger. Eur Heart J. 2015;36(11):65768. DOI: 10.1093/eurheartj/ehu385

30. Okonko DO, Mandal AK, Missouris CG, Poole-Wil- son PA. Disordered iron homeostasis in chronic heart failure: prevalence, predictors, and relation to anemia, exercise capacity, and survival. J Am Coll Cardiol. 2011;58(12):1241-51. DOI: 10.1016/j.jacc.2011.04.040

31. Brownlie Tt, Utermohlen V, Hinton PS, Haas JD. Tissue iron deficiency without anemia impairs adaptation in endurance capacity after aerobic training in previously untrained women. Am J Clin Nutr. 2004;79(3):437-43. DOI: 10.1093/ajen/79.3.437

32. Jankowska EA, Rozentryt P, Witkowska A, Nowak J, Hartmann O, Ponikowska B, et al. Iron deficiency: an ominous sign in patients with systolic chronic heart failure. Eur Heart J. 2010;31(15):1872-80. DOI: 10.1093/ eurheartj/ehq158

33. Jankowska EA, Rozentryt P, Witkowska A, Nowak J, Hartmann O, Ponikowska B, et al. Iron deficiency predicts impaired exercise capacity in patients with systolic chronic heart failure. J Card Fail. 2011;17(11):899906. DOI: $10.1016 / j$.cardfail.2011.08.003

34. Parikh A, Natarajan S, Lipsitz SR, Katz SD. Iron deficiency in community-dwelling US adults with self-reported heart failure in the National Health and Nutrition Examination Survey III: prevalence and associations with anemia and inflammation. Circ Heart Fail. 2011;4(5):599-606. DOI: 10.1161/CIRCHEARTFAILURE.111.960906

35. Corti MC, Guralnik JM, Salive ME, Ferrucci L, Pahor $\mathrm{M}$, Wallace RB, et al. Serum iron level, coronary artery disease, and all-cause mortality in older men and women. Am J Cardiol. 1997;79(2):120-7. DOI: 10.1016/ S0002-9149(96)00697-2

36. Verdon F, Burnand B, Stubi CL, Bonard C, Graff M, Michaud A, et al. Iron supplementation for unexplained fatigue in non-anaemic women: double blind randomised placebo controlled trial. BMJ. 2003;326(7399):1124. DOI: $10.1136 / \mathrm{bmj} .326 .7399 .1124$

37. Cook JD. Diagnosis and management of iron-deficiency anaemia. Best Pract Res Clin Haematol. 2005;18(2):319-32. DOI: 10.1016/j.beha.2004.08.022

38. Skikne BS, Punnonen K, Caldron PH, Bennett MT, Rehu M, Gasior GH, et al. Improved differential diagnosis of anemia of chronic disease and iron deficiency anemia: a prospective multicenter evaluation of soluble transferrin receptor and the sTfR/log ferritin index. Am J Hematol. 2011;86(11):923-7. DOI: 10.1002/ ajh. 22108

39. Beguin Y. The soluble transferrin receptor: biological 
aspects and clinical usefulness as quantitative measure of erythropoiesis. Haematologica. 1992;77(1):1-10.

40. Cotton F, Thiry P, Boeynaems J. Measurement of soluble transferrin receptor by immunoturbidimetry and immunonephelometry. Clin Biochem. 2000;33(4):263-7. DOI: 10.1016/S0009-9120(00)00071-0

41. Pigeon C, Ilyin G, Courselaud B, Leroyer P, Turlin B, Brissot $\mathrm{P}$, et al. A new mouse liver-specific gene, encoding a protein homologous to human antimicrobial peptide hepcidin, is overexpressed during iron overload. J Biol Chem. 2001;276(11):7811-9. DOI: 10.1074/jbc. M008923200

42. Ganz T. Hepcidin, a key regulator of iron metabolism and mediator of anemia of inflammation. Blood. 2003;102(3):783-8. DOI: 10.1182/blood-2003-03-0672

43. Diwan A, Tran T, Misra A, Mann DL. Inflamma- tory mediators and the failing heart: a translational approach. Curr Mol Med. 2003;3(2):161-82. DOI: 10.2174/1566524033361537

44. Konstam MA, Gheorghiade M, Burnett JC, Jr., Grinfeld L, Maggioni AP, Swedberg K, et al. Effects of oral tolvaptan in patients hospitalized for worsening heart failure: the EVEREST Outcome Trial. JAMA. 2007;297(12):1319-31. DOI: 10.1001/ jama.297.12.1319

45. Gheorghiade M, Bohm M, Greene SJ, Fonarow GC, Lewis EF, Zannad F, et al. Effect of aliskiren on postdischarge mortality and heart failure readmissions among patients hospitalized for heart failure: the ASTRONAUT randomized trial. JAMA. 2013;309(11):112535. DOI: $10.1001 /$ jama.2013.1954 